\title{
Recent Studies Assessing the Proliferative Capability of a Novel Adult Stem Cell Identified in Menstrual Blood
}

\author{
Julie G. Allickson ${ }^{*}, 1$, Anthony Sanchez ${ }^{1}$, Natalie Yefimenko ${ }^{1}$, Cesar V. Borlongan ${ }^{2}$ and \\ Paul R. Sanberg ${ }^{2}$ \\ ${ }^{1}$ Cryo-Cell International, Inc., Oldsmar, FL, USA \\ ${ }^{2}$ Center of Excellence for Aging and Brain Repair, Department of Neurosurgery and Brain Repair, University of South \\ Florida, Tampa, FL, USA
}

\begin{abstract}
We are in the beginning of the era of regenerative medicine and many researchers are testing adult stem cells to be used for tissue repair and regeneration in the human body. Many adult stem cells have been discovered since the late 1990 's with more recently a novel adult stem cell described in menstrual blood. The menstrual blood is derived from shedding of the endometrial lining, specifically the functionalis layer, which contains highly proliferative cells used to prepare the female body for implementation of a fertilized egg. Cell characterization experiments of stromal stem cells discovered in menstrual blood have demonstrated cells to be multipotent which can successfully differentiate in vitro into cell lineages derived from the mesoderm and the ectoderm.

When menstrual blood cells were seeded in culture the average number of adherent cells was $8.50 \%$ with a range of $0.48 \%$ to $47.76 \%$. Demonstrating longevity one cell line allowed to grow was subcultured 47 times before complete senescence and death. The menstrual blood stromal stem cell phenotypic analysis incorporates mesenchymal cell markers such as CD13, CD29, CD44, CD49f, CD73, CD90, CD105, CD166, MHC Class I and pluripotent embryonic stem cell markers SSEA-4, Nanog and Oct-4. Karyotypic analysis demonstrated the maintenance of diploid cells without chromosomal abnormalities.

In conclusion preliminary studies have demonstrated menstrual stem cells are easily expandable to clinical relevance. Pivotal pre-clinical studies are now underway to test the safety and efficacy of menstrual stem cells in several different animal models including one for neuroprotection following transplantation into an experimental stroke model. The study demonstrates menstrual stem cells are a novel cell population that may be routinely and safely isolated to provide a renewable source of stem cells from child-bearing women.
\end{abstract}

Keywords: Regenerative medicine, menstrual stromal stem cells, endometrium, allogeneic, pluripotent.

\section{INTRODUCTION}

Stem cells are a source of cells that are capable of renewing over long periods of time through cell division of at least one daughter cell coined long term self renewal. As cells are dividing the new stem cells can remain undifferentiated or become a specific cell type. The cells can become progenitors, a precursor or fully committed cells. Throughout the human body cells are being replenished to replace dying cells or regenerate damaged tissue. Under certain physiological conditions some unspecialized cells may remain dormant until the cells are required. The two main types of stems cells are categorized adult or somatic and embryonic cells. Embryonic stem cells are derived from the inner cell mass of a blastocyst. These cells can divide undifferentiated for an extended period of time and can develop into cells or tissues from all three primary germ layers; mesoderm, endoderm and ectoderm while allowing for the production of

*Address correspondence to this author at the Cryo-Cell International, Inc., Oldsmar, FL, USA; Tel: 813-749-2107; Fax: 813-749-2207; E-mail: jAllickson@cryo-cell.com extraembryonic tissue. These pluripotent cells, if injected into an animal model, can demonstrate the production of teratomas. On the other hand adult stem cells have a controlled behavior and they are multipotent as compared to the embryonic stem cell's pluripotent capacity. Adult stem cells may vary in their capability of differentiation which may be determined by the tissue or organ of origination.

McColloch and Till [1] discussed first the clonal nature of stem cells in bone marrow and Friedenstein plus colleagues $[2,3]$ in the 1970's developed an assay to assess stromal stem cells from bone marrow which they referred to as Colony-Forming-Units-fibroblasts (CFU-f). Caplan [4] followed up by defining the mesengenic process of cell differentiation from mesenchymal lineages. The early work lead to the description of the functional capability of the cell. Prockop [5] discussed the differentiation of non-hematopoietic cells in bone marrow in 1997 . Towards the end of the 1990's Horwitz [6] and colleagues discussed the potential of these stromal cells derived from bone marrow termed mesenchymal cells. Their studies assessed the use of the mesenchymal stem cells from bone marrow for a genetic 
disorder describing a defective osteoclast production in children called Osteogenic Imperfecta. Following this early work others identified highly plastic adult stem cells sources originating from other areas of the body to include adipose tissue, teeth, skin and blood vessels.

\section{ENDOMETRIUM AS A SOURCE OF STEM CELLS}

Endometrial tissue is a prolific source of stem cells that are reoccurring on a monthly basis. Endometrial cells have been studied for many years purporting their stem-like capability associated with regeneration. These cells were harvested directly from the lining of the uterus which has extreme capacity for regeneration after the menstrual cycle. The menstrual cycle which is required for reproduction is controlled by the endocrine system. The cycle spans 28 days starting on the first day of menstruation and includes two phases entitled proliferative and secretory. Stimulated by hormones the menstruation ceases and the lining of the uterus thickens originating from the basalis layer to replenish the functionalis layer shed during menstruation. Under hormonal direction, the endometrium prepares for implantation of an embryo. If implantation does not occur the hormone levels drop and the menses commence which includes a loss of the cells from the functionalis layer of the endometrium. Menstrual blood derived from the uterine tissue also contains highly proliferative cells that support the uterus along with potent growth factor production and cells involved in recurrent angiogenesis.

Some of the first publications in regards to endometrial stem cells were published by Prianishnikov [7] and also by Padykula [8,9] and focused mainly on the epithelial stem cells from the endometrium. Prianishnikov [7] believed cells shed in menstrual blood were non-viable. Gargett and colleagues began studying endometrial cells in the late 1990 's and completed a significant amount of research assessing a population of stem cells residing in the endometrium focused on the basilis layer. In 2005 they studied the putative stem cell activity of both stromal cells and epithelial cells from the endometrium where they selected for the two cell populations post collagenase digestion. They were able to demonstrate that endometrium contains clonogenic cells which do not differ based on the phase of the menstrual cycle [10]. In 2006 Gargett identified and characterized both purified clonal endometrial stromal and clonal epithelial cells from tissue. She was able to determine that these highly purified clonal stem cells were present in less than $0.1 \%$ of the cells [11]. In 2006 she discussed the evidence directed at uterine stem cells and how to assess the functionality of the cell [12]. The human endometrium exhibits dynamic growth during the menstrual cycle expanding approximately $5 \mathrm{~mm}$ in thickness during each cycle following menstruation. Gargett discusses the stem cell geography as historically discussed in the basilis layer as compared to the functionalis or the myometrium [7-9]. Taylor discusses the potential link of endometrial cells to bone marrow stem cells during a study of several HLA-mismatched bone marrow recipients. After an endometrial biopsy he was able to demonstrate a certain percentage of donor cells were identified in the endometrium [13]. There is still a significant amount of data to investigate in the area of endometrial stem cells.

\section{MENSTRUAL BLOOD COLLECTED AS A SOURCE OF STEM CELLS}

Menstrual blood has been studied for its viability and cell characteristics some initial work published in 2008 in Cell Transplantation [14]. The important markers discussed were associated with both adult stem cells as well as embryonic stem cells markers. Some of the multiopotent markers included Oct-4, SSEA-4 and c-kit/CD117. The stromal stem cell fraction of the menstrual blood was able to demonstrate multipotential cell differentiation to five different cell lineages [14]. To be able to characterize the cells a method was developed to procure, transport and process the cells for studies.

Menstrual effluent was procured by use of a silicone medical grade menstrual cup. The collection kit includes two sterile containers loaded with buffered media including heparin and antibiotics, several antiseptic wipes (for cleaning before and after cup is inserted to reduce bioburden), biohazard bags, absorbent material, foam chilling bricks, styrofoam shipping container for maintenance of $1-10^{\circ} \mathrm{C}$ and tube labels. Since we are aware most menstrual cell specimens will have a bioburden level they are treated with antibiotics and keep cold. The collection is scheduled during the heaviest flow of the cycle and the cup will remain in place for no more than 4 hours. To collect the effluent the menstrual cup is inserted into the vagina similarly to the process of inserting a tampon. Once the cells are procured the menstrual cup is removed and the blood is transferred to the sterile media. Optimal transport after collection would be 24 hours and the cells are processed.

The cells were processed with a cocktail of antibiotics (Vancomycin, Cefotaxime sodium, Amikacin, Gentamyiin and Amphotericin B). After the cells were washed in Phosphate Buffered Saline (PBS) they were concentrated and prepared for cryopreservation. The cells were cryopreserved with 10\% Dimethylsulfoxide (DMSO), 30\% PBS, $10 \%$ Human Serum Albumin (25\%) and a 50\% cell suspension. The cellular suspension and the cryopreservative including PBS, Human Serum Albumin (HSA) and DMSO were chilled separately between $2-8^{\circ} \mathrm{C}$ prior to addition. After the cryopreservative was added to the cell suspension the cells were aliquoted into cryovials and cryopreserved in a controlled rate freezer. At a temperature of $-90^{\circ} \mathrm{C}$ the cells were transferred for long term storage to a liquid nitrogen vapor storage freezer held at approximately $-190^{\circ} \mathrm{C}$ until samples were thawed for assessment. To thaw the cells they were removed from the freezer and thawed by agitation in a $37-40^{\circ} \mathrm{C}$ bead bath (Lab Armour, Inc., San Antonio, TX). Just prior to complete thaw the cells were transferred to a wash solution consisting of complete media. The cells were washed twice and seeded in a T-25 tissue culture flask. The cells were grown in Chang's (Irvine Scientific, Santa Ana, CA) Complete Media $[15,16]$. Cells were assessed for time to adherence to flask, growth rate and number of passages. Cells were subcultured by using TrypLE Express (Invitrogen, Carlsbad, CA), washed and replated in complete media.

The cell processing took place in an International Organization for Standardization (ISO) Class 7 Cleanroom in an ISO Class 5 Laminar Flow Hood. Cell processing occurred in a manufacturing line including day one for 
treatment of antibiotics and day two to process and cryopreserve the cells. After the cells were processed they were tested for cell surface markers. They were assessed for a total nucleated cell count and viability tested by 7-aminoactinomycin D (7-AAD) which intercalates into the double stranded DNA of the cell if it is non-viable. Cells were additionally tested for bacteria, fungus, yeast and infectious diseases. Study cells were segregated in a freezer for storage in a bunker with an emergency infrastructure.

\section{CD117 CELL SELECTION}

Stem cells express the antigenic factor CD117 that is also known as a c-kit receptor or stem cell factor receptor. The gene for c-kit encodes a growth factor receptor for Stem Cell Factor (SCF) and plays an important role in hematopoiesis, fertility and melanogenesis. CD117 has been identified in germ cell development, maintenance, and function. Research indicates in the embryonic gonad, CD117 and its ligand SCF, are important for the primordial germ cell survival and proliferation [17]. Additionally, research indicates that CD117 and its corresponding ligand SCF are essential for the gamete production. In other words, CD117 in combination with the ligand SCF are necessary for the survival and proliferation of germ cells of the testis, the spermatogonia, and the oocytes [18-20].

With this level of importance in CD117 and the presence of this cell surface marker on the menstrual blood stromal stem cells after cell expansion, a CD117 positive cell selection was executed. The CD117 stem cells were concentrated by using a MiniMACS separation kit (Miltenyi Biotec, Bergisch Gladbach, Germany) comprising a multistand, MS columns and microbeads used for CD117 cell selection. The MS column was prepared by rinsing with working buffer. The column was placed in a magnetic field providing the collection and concentration of CD117 positive cells. The cells were loaded with the magnet on and the cells were washed several times to collected the CD117 negative fraction. The magnetic field was removed and CD 117 positive cell population was captured in a conical for cell processing. After processing the cells were cryopreserved for future use as previously described [14]. Cells were thawed to expand in culture and the freeze thaw process demonstrated a high level of viable cells with most passages at $100 \%$ viability as determined by 7-AAD. Cells were cultured in Chang's complete media in a T-25 non-treated tissue culture flask. The cells were cultured to isolate adherent cells with non-adherent cells discarded. The cells were subcultured for expansion and analysis.

\section{EXPANSION OF THE MENSTRUAL STROMAL STEM CELLS}

The average total nucleated cell count assessed by a hemocytometer or flow cytometry was 7.9 million with a total viability of approximately $75 \%$ [21]. The cells were tested for longevity in culture. Once the cells were seeded in culture they required 6 to 16 days to attach and become $70 \%$ confluent. When the cells demonstrated $70 \%$ confluency they were subcultured. The first passage generally produced $0.5 \%$ to $22 \%$ adherent cells [21]. The morphology of the adherent cells at passage 2 and passage 27 from the same cell line were identified as fibroblast-like and spindle shaped viewed in Fig. (1).
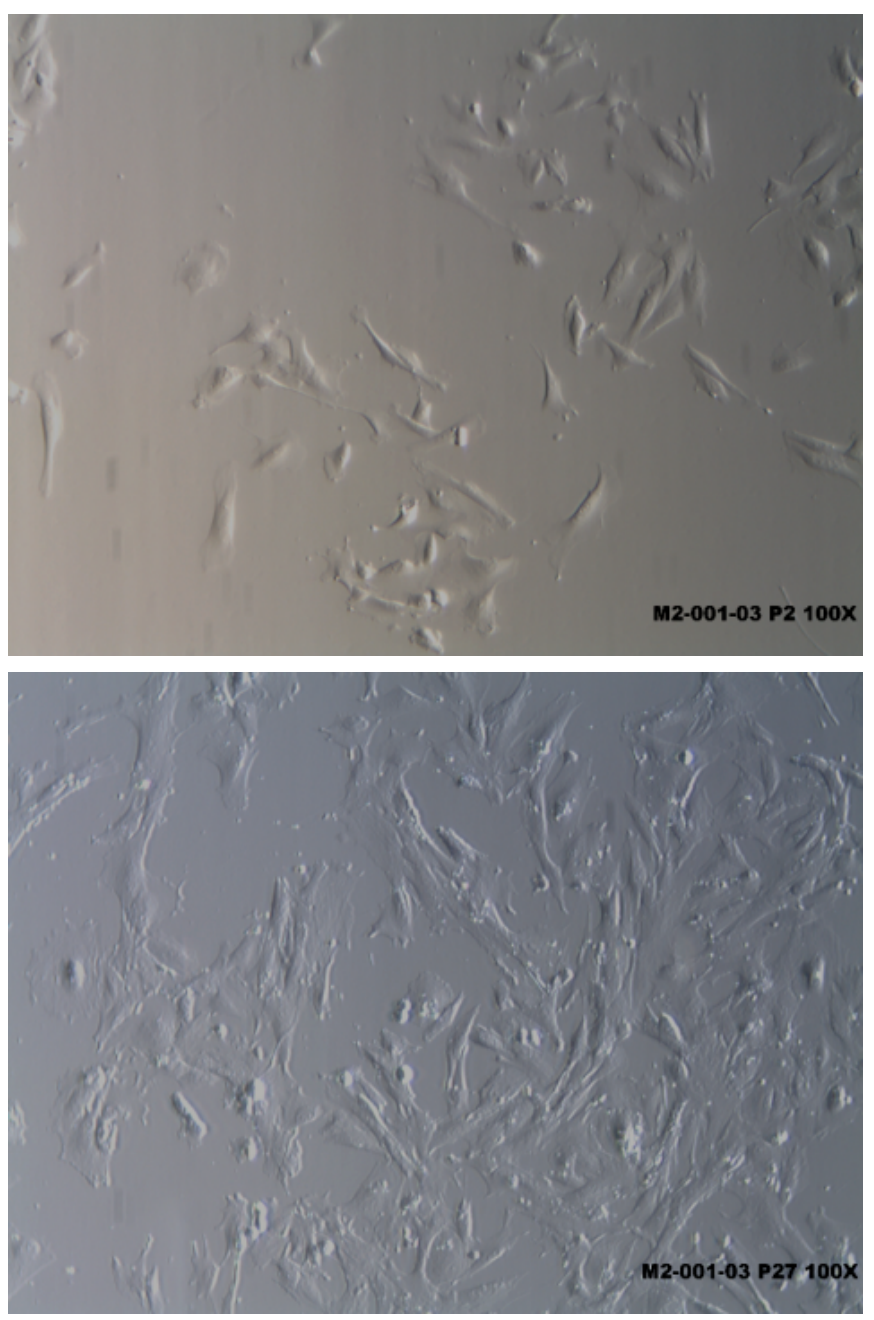

Fig. (1). Adherent spindle shaped menstrual blood stromal stem cells.

In an IRB approved study over 150 samples of menstrual blood were procured to study functional viability. The average number of cells harvested from the menstrual blood in the study was approximately 8 million cells. The cell lines were assessed for the growth rate (total number of cells retrieved from a passage divided by the total number of cells seeded in the previous passage). This resulted in an average growth rate of 68 with a range from 6 to 391. The average number of adherent cells was $8.50 \%$ with a range of $0.48 \%$ to $47.76 \%$ [21]. One cell line allowed to grow until morphological changes were observed and the cells began to senesce was subcultured 47 times before cell death.

\section{ADDITIONAL IN VITRO FUNCTIONAL TESTING ON MENSTRUAL BLOOD STEM CELLS}

The CD117 cell population was expanded, characterized and tested for functional viability in part by cell differentiation to osteogenic, adipogenic, chondrogenic, cardiomyogenic and neurogenic cell differentiation. In summary as described in the past [14] briefly adipogenic differentiation was assessed by using a commercially available differentiation kit (Cambrex East Rutherford, NJ). To assess the 
differentiation cells were stained with Oil Red $\mathrm{O}$ to visualize fat vacuoles which demonstrated $60-70 \%$ differentiation. Cells were tested for osteogenic differentiation and cells were tested by Alzarin Red Staining and for Alkaline Phosphatase Expression by Q-PCR to demonstrate bone mineralization which resulted in $45 \%$ cell differentiation. Chondrogenic differentiation was assessed by staining for sulfated proteoglycans using alcian blue which resulted in $40-50 \%$ differentiation. Neurogenic differentiation was tested by Neurofiliment-3 and Nestin by Q-PCR. They were also tested for Tubulin-III, GFAP (Glial Fibrillary Acidic Protein), MAP-2 and Nestin by IHC staining previously demonstrating neurogenic cell differentiation of $45-50 \%$. The last lineage assessed was cardiogenic differentiation where cells were tested by immunocytochemistry for Actin, Desmin, Troponin and Connexin 43 and demonstrated 50$60 \%$ cell differentiation. The result of the in vitro testing demonstrated the menstrual stromal stem cell has the capacity to be able to differentiate at minimum into cell lineages from the mesoderm and ectoderm.

\section{MENSTRUAL STROMAL STEM CELL MARKERS}

The cell surface markers assessed include: SSEA-4, Oct4, CD117, CD29, CD44. CD166, CD73, CD133, CD90, CD45, CD105 and CD34. Results were assessed by percentage. Positive and negative determination was assessed by greater than $20 \%$ to be labeled positive and less than $20 \%$ to be labeled weak positive or negative (Table 1). Cells were tested by flow cytometry. Menstrual stromal stem cells were able to express not only cell surface markers

Table 1. Summary of Menstrual Stem Cell Markers

\begin{tabular}{|c|c|c|}
\hline Cell Surface Markers & Type & Menstrual Cells \\
\hline \hline SSEA-4 & ESC & Positive \\
\hline Oct-4 & ESC & Positive \\
\hline CD117 & HSC/Germ cell marker & Low positive \\
\hline CD105 & MSC & Positive \\
\hline CD29 & MSC & Positive \\
\hline CD44 & MSC & Positive \\
\hline CD166 & MSC & Positive \\
\hline CD73 & MSC & Positive \\
\hline CD133 & HSC & Negative \\
\hline CD90 & MSC & Positive \\
\hline CD45 & WBC & Negative \\
\hline CD34 & HSC & Negative \\
\hline
\end{tabular}

Adapted table presented: Allickson, J.G. A Novel Multipotent Highly Proliferative Stem Cell Population in Menstrual Blood. Cambridge Healthtech Institute's Molecular Medicine Tri-Conference, March 2008

traditionally observed on mesenchymal or adherent stem cells but also markers associated with embryonic stem cells. The menstrual stem cells have demonstrated its significant telomerase activity of $50 \%$ as compared to human embryonic stem cells at passage 12 and significantly more as com- pared to mouse embryonic fibroblasts as has been demonstrated previously [14]. Other results included karyotype analysis by standard cytogenetic protocol which demonstrated cells had a normal female karyotype [14].

\section{SAFETY STUDIES}

Menstrual blood stromal stem cell samples were assessed by a preliminary General Safety Study in vivo. The safety study assessed cell infusion in Harlan Sprague Dawley Mice and Dunkin Hartley Albino Guinea Pigs. The study participants received an intraperitoneal injection of the cells or the negative control material. The participants were observed for a seven day period. After one week the participants were assessed for weight, survival and specific response to the cells. Under the conditions of the study the presence of extraneous toxic contaminants was not detected and all animals remained healthy, without reactions and no weight loss.

\section{ALLOGENEIC RESPONSE}

Menstrual blood stem cells were tested for allogeneic response in culture with peripheral blood mononuclear cells (PBMN). The stem cells were analyzed at a 1:2 stimulator (mitomycin $\mathrm{C}$ treated $\mathrm{PBMN}$ ) to responder cell (menstrual blood stem cells) ratio for 6 days. The menstrual blood stem cells demonstrated a moderately weak stimulatory response in a mixed lymphocyte reaction (MLR) (Table 2). An emerging bank of data allows for the classification of the mesenchymal stem cell from bone marrow as immunosuppressive cell source derived from studies with human cells [22, 23].

Table 2. Menstrual Blood Stem Cells Mixed Lymphocyte Response to Human PBMC

\begin{tabular}{|c|c|c|}
\hline \multirow{2}{*}{ Stimulator } & \multicolumn{2}{|c|}{ Stimulation index of Responder $^{\mathbf{b}}$} \\
\cline { 2 - 3 } & A PBMC & B PBMC \\
\hline \hline positive control PBMC & 9.2 & 6.5 \\
\hline M2 M202C cells & 4.7 & 3.8 \\
\hline M2 M203E cells & 3.0 & 3.6 \\
\hline M2 M201 cells & 3.5 & 2.3 \\
\hline
\end{tabular}

Stimulator cells were mitomycin $\mathrm{C}$ treated stem cell lines or heterologous human PBMC

${ }^{\mathrm{b}}$ Responder cells were heterologous human PBMC

\section{INTERPRETATIONS}

For responder PBMC A, a stimulation index of $\geq 3$ is 2 standard deviations over background

For responder PBMC B, a stimulation index of $\geq 2.5$ is 2 standard deviations over background

These values can be used as cut-off values, above which could be considered as significant induction of MLR

\section{ASSESSING THE NEED FOR CELLULAR THERAPY IN STROKE}

Laboratory investigations have explored the functional capacity of the multipotent menstrual stromal stem cells in a 
rat stroke model assessing efficacy as a graft source for stroke therapy. In the United States of America stroke is the third leading cause of death therefore allowing a significant opportunity to therapeutically treat the disease. Approximately 150,000 people die each year in the US from stroke and approximately 800,000 people suffer a stroke per year.

Currently Tissue Plasminogen Activator (TPA) is the only know FDA approved treatment for stroke. TPA is a thrombolytic agent for the dissipation of clots. The drug is approved in the United States of America for acute treatment of ischemic stroke. Unfortunately the drug is time sensitive and must be administered within hours of the attack to be effective and the earlier the stroke is treated the better for safety and functional outcome [24]. This treatment is not without risks as studies demonstrate increased risk of intracranial hemorrhage in patients with a severe neurological deficient. Generally studies observed 1 out of 100 with a severe baseline deficit that demonstrates a negative outcome after treatment with TPA [25].

In the past a significant amount of work has been done to assess adult stem cells specifically cord blood as a treatment modality in a middle cerebral artery occlusion (MCAo) rat model for stroke. Sanberg and colleagues have published numerous articles studying the effects of cord blood stem cells in a stroke model [26-32]. Results collectively demonstrate brain repair in this model which results from neurotrophic responses produced by the cell infusion that may alter cell survival, angiogenesis and provide an anti-inflammatory reaction to nurture the benefits of cellular treatment in stroke.

In light of the gap in therapeutic options for stroke and the early results assessing cord blood stem cells in stroke a study assessed menstrual blood stromal stem cells due to the immunological naive and highly proliferative nature of the cells.

\section{ASSESSMENT OF PRE-CLINICAL RAT STROKE MODEL}

A recent report has revealed the potential of menstrual blood stromal stem cells for its therapeutic benefits in a rat stroke model [33]. Initially cells were characterized via immunocytochemical assays for additional markers. Testing revealed markers to include: Nanog an embryonic stem cell marker and neuronal markers; Nestin, MAP-3, GFAP, NeuN when cultured in neuronal media. Additional testing provided confirmation of markers Oct-4, SSEA and CXCR4, a stem cell chemotaxis marker [33]. Cells were tested in vitro by co-culture, testing cell supernatant and in vivo in the MCAo model. Co-cultured cells as well as conditioned media provided positive results demonstrating reduced cell death and improved cell survival when tested with primary neurons that were Oxygen Glucose Deprived. The MCAo model was treated with menstrual stromal stem cells by intravenous (IV) and intracerebral (IC) injection and was evaluated post necropsy for engraftment and cell differentiation. Prior to necropsy the patients were studied for behavioral effects of the treatment. Compared to a vehicle control the rat model exhibited significant protection against ischemic cell death. Evaluation of potential neurotrophic factors associated with repair was performed with in vitro studies revealing elevated levels of trophic factors, such as VEGF, BDNF, and NT-3, in the media of OGD-exposed neurons cultured menstrual blood stromal stem cells. Besides the demonstration of efficacy created by the proposed cell bystander effect resulting in trophic factors, cells were also identified in the brain of the MCAo model. Additionally results demonstrated no Graft $v s$. Host Disease in the xenogeneic animal model without immunosuppression coupled to the benefit of no tumor formation from the injection of a stem cell population retaining embryonic stem cell markers. The results of this study provide positive results for a novel cell source that may be tested in the future for treatment of stroke.

\section{ON-GOING RESEARCH STUDIES}

Other functional studies that are being assessed with the menstrual blood stem cells include a breast cancer model studying the homing capability of the cell for its possible use as a vehicle for a chemotherapeutic agent. A blood vessel formation model which may be the stepping stone to treat a variety of disorders. A diabetic streptozotocin mouse model which is improved at a time when more than 30 million people in the US are diagnosed with the disease and with India and China exceeding these numbers. Wound healing is important especially in a scenario such as diabetic ulcers where there is a risk for amputation associated with the ischemia. Other studies include urinary incontinence and the early diagnosis of endometriosis a disease causing anguish for many women during child bearing years. Researchers are intrigued to study an adult stem cell that demonstrates longevity in culture, maintenance of embryonic stem cell markers, efficient growth rate, low immunological potential, production of tropic factors and no initial signs of tumorgenicity.

\section{SUMMARY}

Menstrual blood stromal stem cells demonstrate extreme potential as a source in cellular therapeutic intervention. Menstrual stem cells are an attractive cell source as displayed by their longevity in culture with most stem cell sources being subcultured 10 or 20 passages. Studies have demonstrated the cells retain a normal karyotype when subcultured out 12 times and additionally that cells retain embryonic stem cell markers even after infusion into the brain of a rat stroke model or in culture for up to 20 passages. The cells demonstrated safety in 2 different animal models. The cells are currently being assessed in many different applications as an autologous source of cells but from the initial pilot studies on the immunological potential it appears to be an immunologically naive source. The cells demonstrate an immaturity denoted by the embryonic stem cell markers coupled to lack of evidence to date of tumor production compared to embryonic stem cells that require harnessing the cellular potential. Embryonic stem cells currently cannot be used in an undifferentiated state but must become a specialized cell to harness the uncontrollable proliferative capacity unlike the menstrual stem cell.

Interestingly menstrual blood stem cells can be available from woman during child-bearing years and potentially up to 12 times a year which allows ample time to be able to store 
the cells for potentially multiple therapies. Highly important in the $21^{\text {st }}$ century would be procurement executed in a noninvasive fashion as compared to bone marrow which requires an operating room and general anesthesia. The cells are not only highly plastic but very hearty as they may be processed up to 96 hours after collection which still allows for the provision of an expansive cell source in culture. In theory we observe menstrual stem cells where umbilical cord blood stem cells were over 20 years ago beginning with basic research and pre-clinical studies assessing the cells proliferative capacity in vitro and in vivo. Menstrual blood stromal stem cells have an opportunity to be a major player in regenerative medicine due to its plasticity and longevity which demonstrates great promise for its future use in tissue repair, treatment of disease and possibly cosmeceutical treatments which is highly coveted in the $21^{\text {st }}$ century.

\section{DISCLOSURE}

PRS is a shareholder of Cryo-Cell International Inc. and Co-Founder of Saneron CCEL Therapeutics Inc. CVB is a consultant for Saneron. JGA is an employee of Cryo-Cell International, owns stock in the company and is a coinventor of the patent describing the menstrual blood stem cell technology.

\section{ACKNOWLEDGEMENTS}

We would like to thank Cryo-Cell International Inc. for providing the cells which established the basis for these research investigations. We would like to acknowledge Mercedes Walton, COB/CEO of Cryo-Cell International Inc. for her hypothesis related to the potential to procure, process and cryopreserve menstrual stem cells which established the basis for this research study. Cryo-Cell International has filed patent applications associated with intellectual property related to the processes; methodologies and composition of matter referenced in this study and Saneron CCEL Therapeutics and Cryo-Cell International Inc. has filed a patent application associated with intellectual property of the rat stroke model treated with menstrual stem cells. We thank the teams from Cryo-Cell International Inc. for cell processing and testing, Center of Excellence for Aging \& Brain Repair, Department of Neurosurgery and Brain Repair, University of South Florida and Saneron CCEL Therapeutics Inc. for the work on the stroke model, Marin Biologic Laboratories (Tiburon, CA) for performing studies associated with Mixed Lymphocyte Reactions and Willa Myers for her assistance in word processing.

\section{REFERENCES}

[1] Becker AJ, McCulloch EA, Till JE. Cytological demonstration of the clonal nature of spleen colonies derived from transplanted mouse marrow cells. Nature 1963; 197: 452-4.

[2] Friedenstein AJ, Deriglasova UF, Kulagina NN, et al. Precursors for fibroblasts in different populations of hematopoietic cells as detected by the in vitro colony assay method. Exp Hematol 1974; 2: 83-92.

[3] Friedenstein AJ, Chailakhyan RK, Latsinik NV, Panasyuk AF, Keiliss-Borok IV. Stromal cells responsible for transferring the microenvironment of the hematopoietic tissues: cloning in vitro and retransplantation in vivo. Transplantation 1974; 17: 331-40.
[4] Caplan AI. The mesengenic process. Clin Plast Surg 1994; 21: 42935.

[5] Prockop DJ. Marrow stromal cells as stem cells for nonhematopoietic tissues. Science 1997; 276: 71-4.

[6] Horwitz EM, Prockop DJ, Fitzpatrick LA, et al. Transplantability and therapeutic effects of bone marrow-derived mesenchymal cells in children with osteogenesis imperfecta. Nat Med 1999; 5: 309-13.

[7] Prianishnikov VA. On the concept of stem cell and a model of functional-morphological structure of the endometrium. Contraception 1978; 18: 213-23.

[8] Padykula HA, Coles LG, McCracken JA, King NW Jr, Longcope C, Kaiserman-Abramof IR. A zonal pattern of cell proliferation and differentiation in the rhesus endometrium during the estrogen surge. Biol Reprod 1984; 31: 1103-18.

[9] Padykula HA, Coles LG, Okulicz WC, Rapaport SI, McCracken JA, King NW Jr. The basalis of the primate endometrium: a bifunctional germinal compartment. Biol Reprod 1989; 40: 681-90.

[10] Schwab KE, Chan RW, Gargett CE. Putative stem cell activity of human endometrial epithelial and stromal cells during the menstrual cycle. Fertil Steril 2005; 84(Suppl 2): 1124-30.

[11] Gargett CE. Identification and characterization of human endometrial stem/progenitor cells. Aust N Z J Obstet Gynaecol 2006; 46: 250-53.

[12] Gargett CE. Uterine stem cells: what is the evidence? Hum Reprod Update 2007; 13: 87-101.

[13] Taylor HS. Endometrial cells derived from donor stem cells in bone marrow transplant recipients. JAMA 2004; 292: 81-5.

[14] Patel AN, Park E, Kuzman M, Benetti F, Silva FJ, Allickson JG. Multipotent menstrual blood stromal stem cells: isolation, characterization and differentiation. Cell Transplant 2008; 17: 30311.

[15] Chang HC, Jones OW, Masui H. Human amniotic fluid cells grown in a hormone-supplemented medium: suitability for prenatal diagnosis. Proc Natl Acad Sci USA 1982; 79: 4795-99.

[16] Delo DM, De Coppi P, Bartsch G Jr, Atala A. Amniotic fluid and placental stem cells. Methods Enzymol 2006; 419: 426-38.

[17] Orr-Urteger A, Avivi A, Zimmer Y, Givol D, Yarden Y, Lonai P. Department of chemical immunology, Weizmann Institute of Science, Rehovot, Israel. Development 1990; 109: 911-23.

[18] Sorrentino V, Giorgi M, Geremia R, Besmer P, Rossi P. Expression of the c-kit proto-oncogene in the murine male germ cells. Oncogene 1991; 6: 149-51.

[19] Horie K, Takakura K, Taii S, et al. The expression of c-kit protein during oogenesis and early embryonic development. Biol Reprod 1991; 45: 547-52.

[20] Hoyer PE, Byskov AG, Møllgård K. Stem cell factor and c-Kit in human primordial germ cells and fetal ovaries. Mol Cell Endocrinol 2005; 234(1-2):1-10.

[21] Allickson, JG. Menstrual blood stromal cells demonstrate a multipotent proliferative capacity in culture. Cytotherapy 2008; 10(Suppl 1): 221.

[22] Tse WT, Pendleton JD, Beyer WM, Egalka MC, Guinan EC. Suppression of allogeneic T-cell proliferation by human marrow stromal cells: implications in transplantation. Transplantation 2003; 75: 389-97.

[23] Kiss J, Urban VS, Dudics V, Vas V, Uher F. Mesenchymal stem cells and the immune system--immunosuppression without drugs? Orv Hetil 2008; 149: 339-46.

[24] Bluhmki E, Chamorro A, Dávalos A, et al. Stroke treatment with alteplase given $3 \cdot 0-4 \cdot 5 \mathrm{~h}$ after onset of acute ischemic stroke (ECASS III): additional outcomes and subgroup analysis of a randomized controlled trial. Lancet Neurol 2009; 8: 1095-102.

[25] Saver JL. Hemorrhage after thrombolytic therapy for stroke: the clinically relevant number needed to harm. Stroke 2007; 38: 227983.

[26] Chen J, Sanberg PR, Li Y, et al. Intravenous administration of human umbilical cord blood reduces behavioral deficits after stroke in rats. Stroke 2001; 32: 2682-8.

[27] Lu D, Sanberg PR, Mahmood A, et al. Intravenous administration of human umbilical cord blood reduces neurological deficit in the rat after traumatic brain injury. Cell Transplant 2002; 11:275-81.

[28] Zigova T, Song S, Willing AE, et al. Human umbilical cord blood cells express neural antigens after transplantation into the developing rat brain. Cell Transplant 2002; 11: 265-74. 
[29] Willing $\mathrm{AE}$, Lixian J, Milliken $\mathrm{M}$, et al. Intravenous versus intrastriatal cord blood administration in a rodent model of stroke. Neurosci Res 2003; 73: 296-307.

[30] Newman MB, Willing AE, Manresa JJ, Davis-Sanberg C, Sanberg PR. Stroke-induced migration of human umbilical cord blood cells: time course and cytokines. Stem Cells Dev 2005; 14: 576-86.

[31] Vendrame M, Gemma C, de Mesquita D, et al. Anti-inflammatory effects of human cord blood cells in a rat model of stroke. Stem Cells Dev 2005; 14: 595-604.
[32] Park DH, Borlongan CV, Willing AE, et al. Human umbilical cord blood cell grafts for brain ischemia. Cell Transplant 2009; 18: 98598.

[33] Borlongan CV, Kaneko Y, Maki M, et al. Menstrual blood cells display stem cell-like phenotypic markers and exert neuroprotection following transplantation in experimental stroke. Stem Cells Dev 2010; 19: 439-52.

(C) Allickson et al.; Licensee Bentham Open.

This is an open access article licensed under the terms of the Creative Commons Attribution Non-Commercial License (http://creativecommons.org/licenses/by$\mathrm{nc} / 3.0 /$ ), which permits unrestricted, non-commercial use, distribution and reproduction in any medium, provided the work is properly cited. 